\title{
Integral display for non-static observers
}

\author{
Adrian Dorado, Seokmin Hong, Genaro Saavedra, and Manuel Martinez-Corral \\ Department of Optics, University of Valencia, E-46100 Burjassot, Spain. \\ Adrian.Dorado@uv.es, Seokmin.Hong@uv.es, Genaro.Saavedra@uv.es, Manuel.Martinez@uv.es \\ B. Javidi \\ Electrical and Computer Engineering Dept., University of Connecticut, Storrs, CT 06269-2157 \\ bahram@engr.uconn.edu
}

\begin{abstract}
We propose to combine the Kinect and the Integral-Imaging technologies for the implementation of Integral Display. The Kinect device permits the determination, in real time, of $(\mathrm{x}, \mathrm{y}, \mathrm{z})$ position of the observer relative to the monitor. Due to the active condition of its IR technology, the Kinect provides the observer position even in dark environments. On the other hand, SPOC 2.0 algorithm permits to calculate microimages adapted to the observer 3D position. The smart combination of these two concepts permits the implementation, for the first time we believe, of an Integral Display that provides the observer with color 3D images of real scenes that are viewed with full parallax and which are adapted dynamically to its $3 \mathrm{D}$ position.
\end{abstract}

Key words: 3D display, Integral Imaging, Eye-tracking, Kinect.

Integral Imaging (InI) is a technique whose origins go back to Lippmann who, in 1908, proposed Integral photography [1]. Integral Photography is an autostereoscopic technique that permits to record and display the spatio-angular information of a 3D scene. InI has greatly evolved since Lippmann first proposal and, nowadays, numerous research groups work in the topic trying to improve several aspects of the technique. There are new capturing methods, as [2], and novel ways to process the recorded spatio-angular information [3]. In addition, there has been several implementations of InI monitors [4], [5], as well as some proposals to combine InI technology with other techniques. As, for example, combining InI with eyetracking [6], [7] and 3D microscopy [8], [9].

In InI, a 3D image is reconstructed using an InI monitor which, usually, is composed by a high resolution display and a microlens array. The 3D information can be displayed in the form of elemental images or microimages [5] but, on this work, we will only consider microimages. In order to display a good 3D image, each microimage needs to fit under one microlens. Finally, the light coming from the displayed microimages is integrated in the 3D space, reconstructing the 3D scene, see Figure 1.

The position of an observer with respect the InI monitor is important because the area where the displayed 3D image can be observed correctly is limited. The viewing area and viewing angle of the InI monitor is mainly determined by the lens size, the number of lenses and the distance between the pixels and the lenses [10], see Figure 2. An observer looking at the InI monitor inside of the viewing area will see a 3D reconstructed image with full parallax. But, if the observer looks at the InI monitor outside of the viewing area, light coming from pixels of incorrect microimages will be seen, producing the undesired flipping effect, in which the observer will see an image with artifacts. 


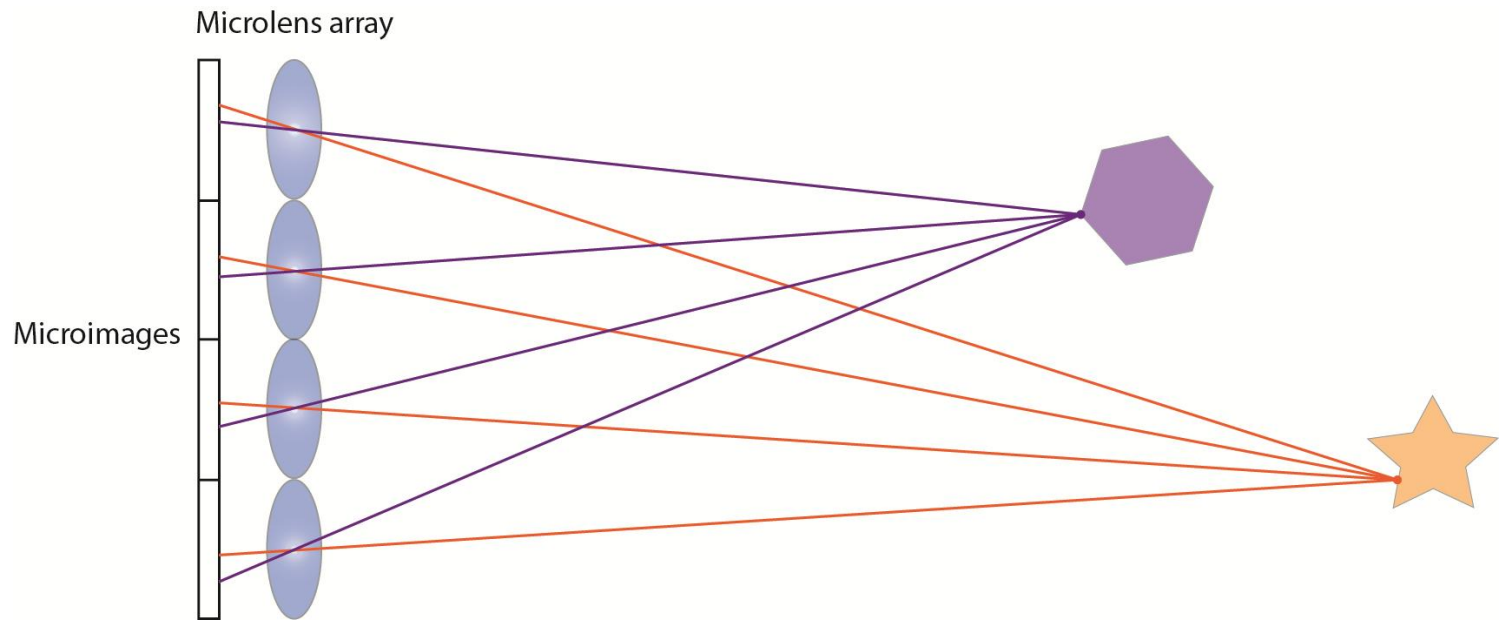

Figure 13D reconstruction of a 3D scene via light integration using an InI monitor.

The Kinect 2 is a device that has a high resolution RGB camera and an infrared time of flight system that emits IR flashes at high frequency. The IR light emitted is reflected on the surfaces in front of the Kinect 2 , and the sensor evaluates the depth distance of those surfaces by measuring the light's returning time [11], [12]. Another remarkable trait of this device is that, thanks to its infrared technology, the Kinect 2 can work in dark environments. In addition, Kinect 2 can be used to track the 3D position of an observer. With the SDK developed by Microsoft it is possible to track the lateral position of the head of the observer and, using the infrared time of flight technology, by adding depth information it is possible to obtain the $3 \mathrm{D}$ position of the observer.

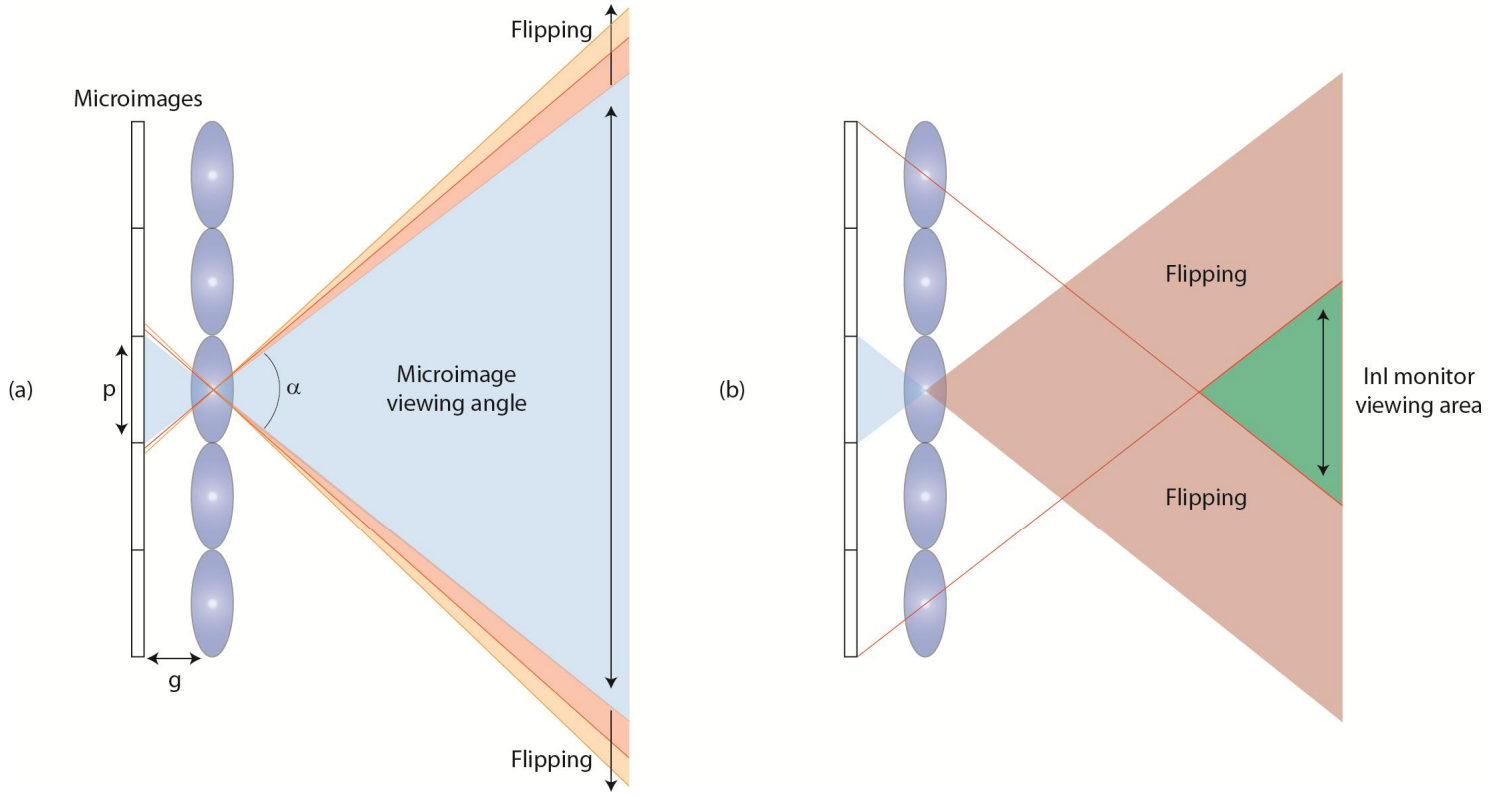

Figure 2 Viewing area of an InI system: (a) shows the viewing angle of just one lens. When an observer watches light that came from an incorrect microimage, the flipping effect occurs; (b) shows the viewing area, taking into account the whole InI monitor; additional lenses reduces the viewing area of the InI monitor. 
Our proposal is to implement an Integral Display that provides the observer with color 3D images of real scenes that are viewed with full parallax and which are adapted dynamically to its $3 \mathrm{D}$ position. We use a Kinect 2 device to track the 3D position of the observer, even in dark environments. We capture the 3D scene using the synthetic aperture method [13] and we adapt the image to the InI monitor using the SPOC 2.0 algorithm [5]. When the observer moves laterally in front of the InI monitor, we shift the displayed microimages and, therefore, the viewing area of the InI monitor increases by avoiding the flipping effect, see Figure 3. But, in order to not lose the 3D parallax, the pixel that the observer is watching is changed each time the images moves. In addition, we also change the reference plane of the displayed image when the observer moves its position orthogonally with respect the position of the InI monitor.

(a)

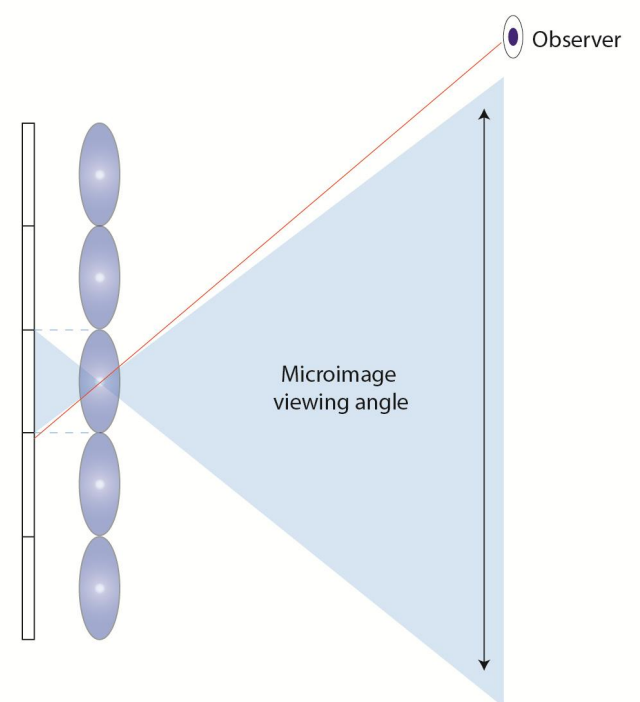

(b)

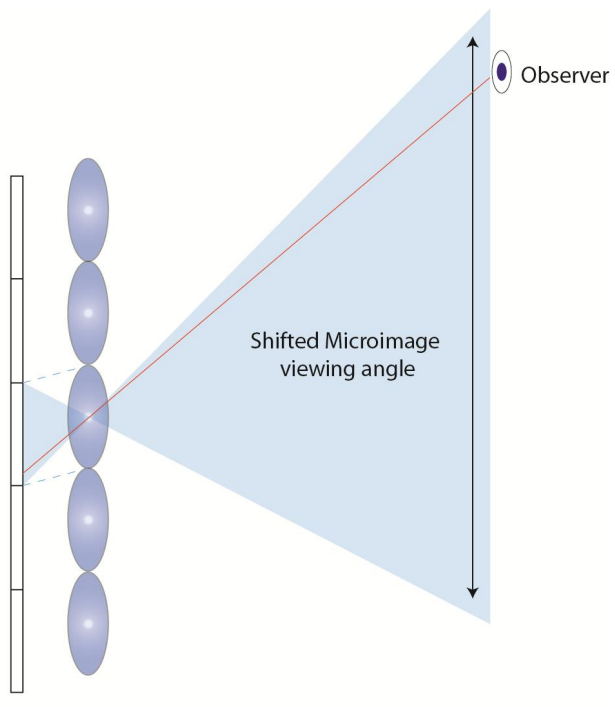

Figure 3 In (a), the observer is outside the microimage viewing angle and, therefore, sees the flipping effect. In (b), after shifting the microimages, the observer sees the correct microimage through the correct microlens.

To prove this concept, we have captured an Integral Image with a Canon 450D camera which position is controlled by motors. Also, we have performed an InI monitor composed by a 4K Dell-P2415Q screen with 3840x2160 pixels and a microlens array with microlenses with focal length, $\mathrm{f}=8 \mathrm{~mm}$, and pitch, $\mathrm{p}=$ $1.6 \mathrm{~mm}$. In order to adapt the captured Integral Image to the InI monitor we have applied the SPOC 2.0 algorithm, that permits us to choose the field of view and the reference plane of the displayed microimages at will [5]. To perform our experiment, we have generated two sets of 23 images, each set with a different position of the reference plane. On each set, one image is chosen as the central one and the rest are shifted an $x$ number of pixels, where $x$ is the relative position of the image with respect the central one. The direction the microimages are shifted is indicated by the position of the image with respect the central one, to the right if it is on the right of the central one and to the left if it is on the left.

In our experiment, the observer is simulated by a camera that shifts its positions. The total lateral movement is from 0 to $300 \mathrm{~mm}$. The number of microimages of the displayed image for this experiment are $118 \times 118$, where each microimage have $11.75 \times 11.75$ pixels each. When the observer moves in front of the InI monitor, the Kinect 2 detects the shift in position of the viewer and changes the displayed image depending on the current 3D position of the observer, see Figure 4 and Figure 5. In Figure 6 we also demonstrate that this system works in dark environments. 


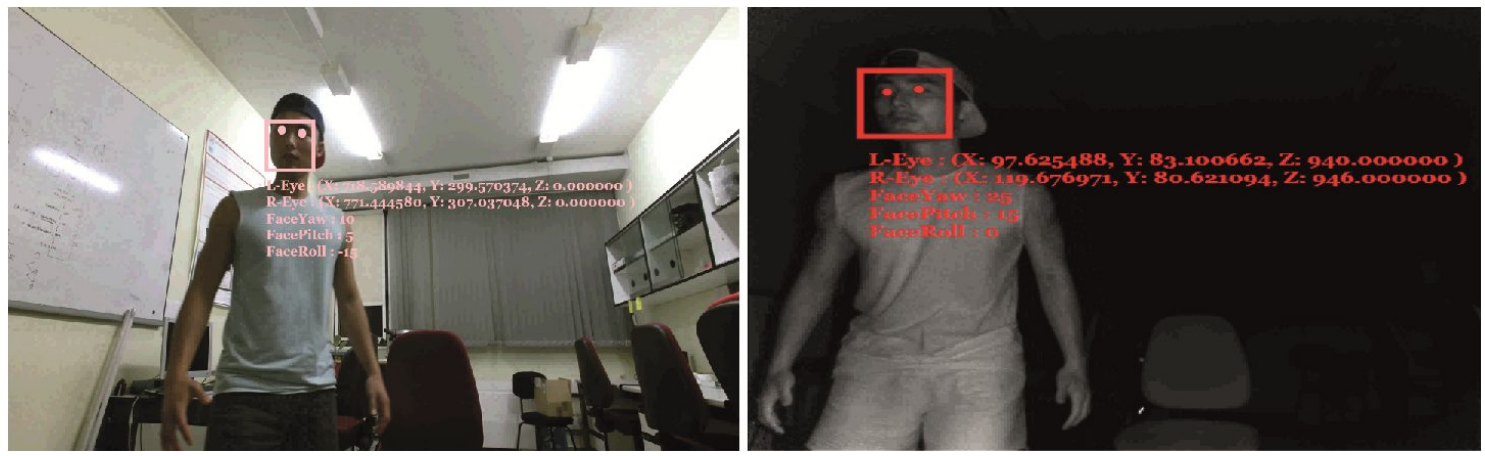

Figure 4 The Kinect 2 is a device that permits to track the 3D position of an observer. It uses infrared technology, so it works in dark environments.

(a)

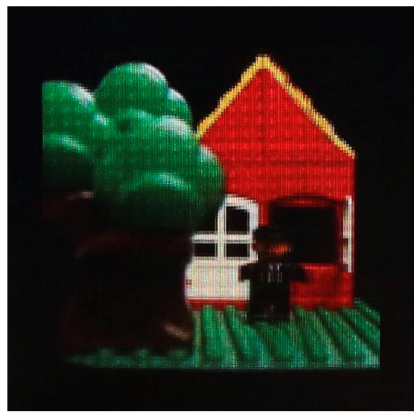

(b)
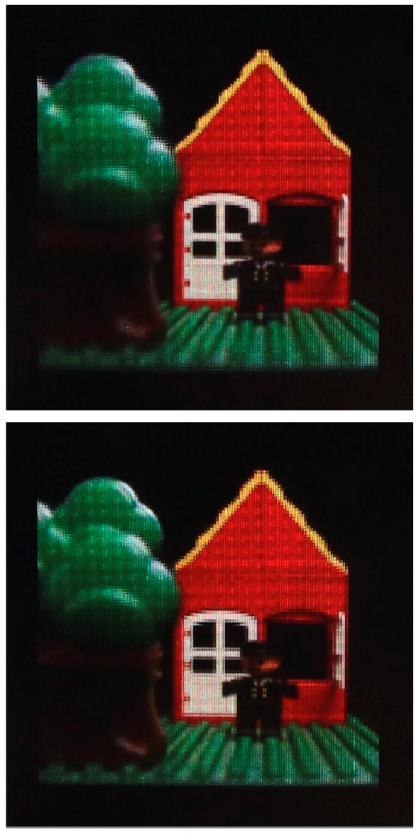

$\mathrm{x}=0 \mathrm{~mm}$
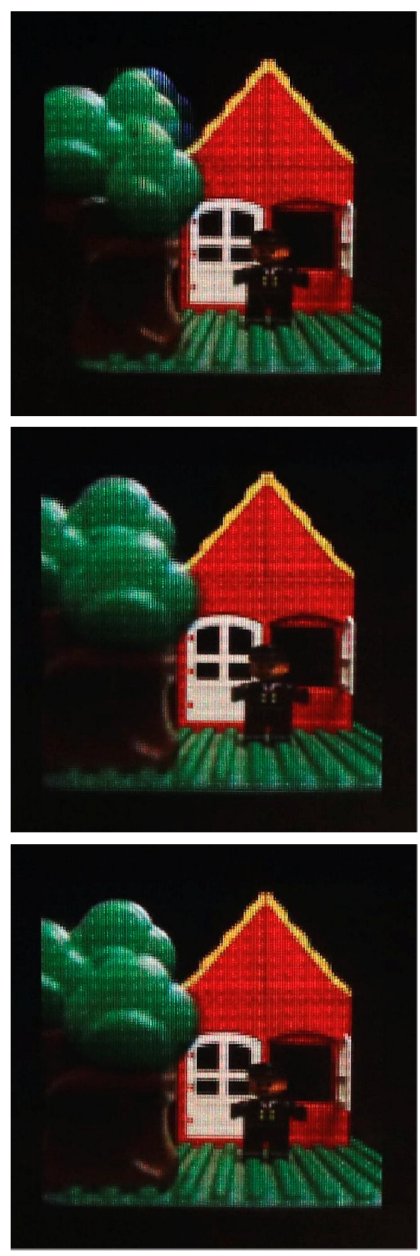

$\mathrm{x}=75 \mathrm{~mm}$
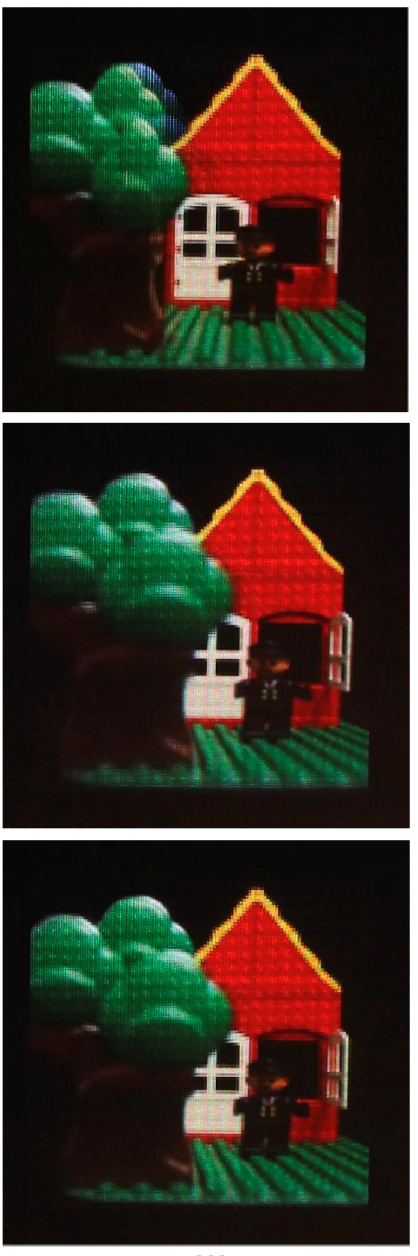

$x=300 \mathrm{~mm}$

Figure 5 Images captured in different positions in front of the InI monitor. The camera was shifted laterally from $x=0$ to $x=300 \mathrm{~mm}$. (a) Shows three different positions when the tracking is not activated. The expected results of watching an InI monitor: outside of the viewing area the flipping effect appears; (b) and (c) shows the same positions when the tracking is activated, there is no flipping and the viewing area is greatly increased. In (b) the image shown is from the first set generated, which has the reference plane near the house and in (c) the image shown is from the second set, which has the reference plane near the doll. 

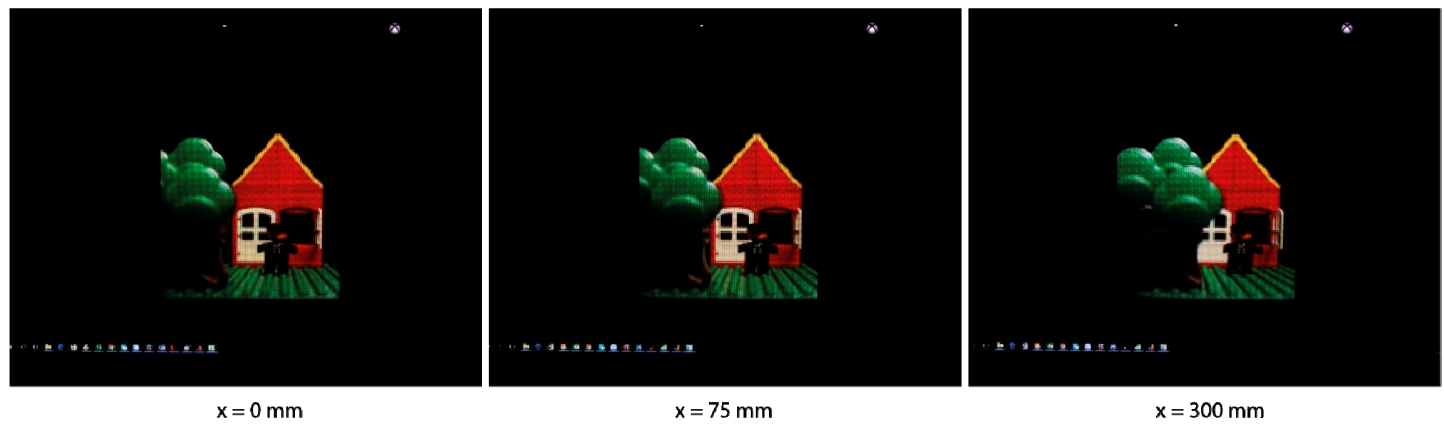

Figure 6 Images captured in different positions in front of the InI monitor. The InI monitor was in dark environment. The camera was shifted laterally from $\mathrm{x}=0$ to $\mathrm{x}=300 \mathrm{~mm}$. The reference plane is placed near the doll.

Summarizing, we have implemented an InI monitor that is able to display 3D images adapted to the $3 \mathrm{D}$ position of the observer and that it can work in dark environments. This is possible thanks to the combination of the SPOC 2.0 algorithm and a Kinect 2 device.

\section{ACKNOWLEDGEMENTS}

This work was supported by the Ministerio de Economía y Competitividad, Spain (Grant No. DPI 2015 66458-C2- 1-R), and by Generalitat Valenciana, Spain (project PROMETEOII/2014/072). A. Dorado acknowledges a predoctoral fellowship from the MINECO (BES-2013-064867). S. Hong acknowledges a predoctoral grant from University of Valencia (UV-INVPREDOC15-265754).

\section{REFERENCES}

[1] G. Lippmann, "Epreuves reversibles donnant la sensation du relief," J. Phys. 7, 821-825 (1908).

[2] S. Hong, D. Shin, B.-G. Lee, A. Dorado, G. Saavedra, and M. Martínez-Corral, "Towards 3D television through fusion of Kinect and Integral-Imaging concepts” J. Display Technol. 11, 894-899 (2015).

[3] S. Wanner and B. Goldluecke, "Globally Consistent Depth Labeling of 4D Light Fields," Proc. IEEE Conf. Computer Vision and Pattern Recognition (CVPR), 41-48 (2012).

[4] J. Arai, M. Kawakita, T. Yamashita, H. Sasaki, "Integral three-dimensional television with video system using pixel-offset method" Opt. Express 11, 3474-85 (2013).

[5] M. Martinez-Corral, A. Dorado, H. Navarro, G. Saavedra, and B. Javidi, "3D display by Smart Pseudoscopic-to-Orthoscopic Conversion with tunable focus" Appl. Opt. 53, 19-26 (2014).

[6] X. Shen, M. Martinez-Corral, and B. Javidi, "Head Tracking Three-Dimensional Integral Imaging Display Using Smart Pseudoscopic-to-Orthoscopic Conversion” J. Display Technol. 12, 542-548 (2016).

[7] S. Hong, D. Shin, J.J Lee, and B.-G Lee, "Viewing Angle-Improved 3D Integral Imaging Display with Eye Tracking Sensor,” J. lnf. Commun. Converg. Eng. 12, 208-214 (2014).

[8] M. Levoy, R. Ng, A. Adams, M. Footer, and M. Horowitz, “Light field microscopy,” ACM Trans. Graph. 25(3), 924-934 (2006). 
[9] A. Llavador, E. Sánchez-Ortiga, J.C. Barreiro, G. Saavedra, and M. Martínez-Corral, "Resolution enhancement in integral microscopy by physical interpolation," Biomed. Opt. Express 6, 2854-2863 (2015).

[10] H. Deng, Q.-H. Wang, L. Li and D.-H. Li, "An integral-imaging three-dimensional display with wide viewing angle," J Soc Inf Disp. 19, 679-684 (2011)

[11]D. Pagliari et al., "Calibration of Kinect for Xbox one and comparison between the two generations of Microsoft sensors," Sensors 15, 27569- 27589 (2015).

[12]R. Smeenk, "Kinect v1 and Kinect v2 fields of view compared," http://smeenk.com/kinect-field-ofview-comparison. (2014)

[13] S. Jang and B. Javidi, “Three-dimensional synthetic aperture integral imaging” Opt. Lett. 27, 11441146 (2002). 